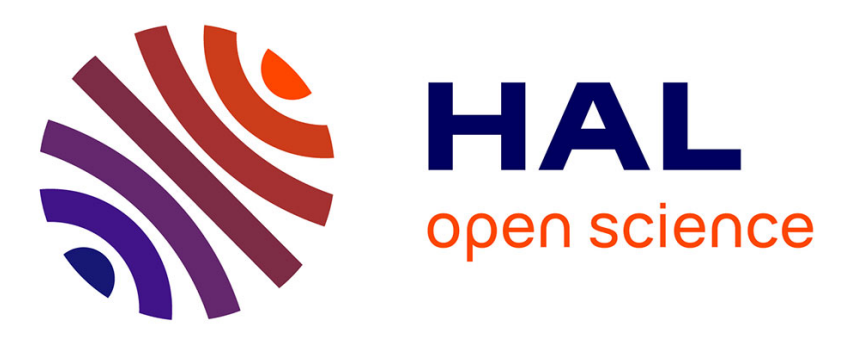

\title{
Hypernetted-chain investigation of the random first-order transition of a Lennard-Jones liquid to an ideal glass
}

Jean-Marc Bomont, Jean-Pierre Hansen, Giorgio Pastore

\section{To cite this version:}

Jean-Marc Bomont, Jean-Pierre Hansen, Giorgio Pastore. Hypernetted-chain investigation of the random first-order transition of a Lennard-Jones liquid to an ideal glass. Physical Review E: Statistical, Nonlinear, and Soft Matter Physics, 2015, 92 (4), pp.042316. 10.1103/PhysRevE.92.042316 . hal-01515452

\author{
HAL Id: hal-01515452 \\ https://hal.univ-lorraine.fr/hal-01515452
}

Submitted on 11 May 2017

HAL is a multi-disciplinary open access archive for the deposit and dissemination of scientific research documents, whether they are published or not. The documents may come from teaching and research institutions in France or abroad, or from public or private research centers.
L'archive ouverte pluridisciplinaire HAL, est destinée au dépôt et à la diffusion de documents scientifiques de niveau recherche, publiés ou non, émanant des établissements d'enseignement et de recherche français ou étrangers, des laboratoires publics ou privés. 


\title{
Hypernetted-chain investigation of the random first-order transition of a Lennard-Jones liquid to an ideal glass
}

\author{
Jean-Marc Bomont, ${ }^{1, *}$ Jean-Pierre Hansen, ${ }^{2,3, \dagger}$ and Giorgio Pastore ${ }^{4, \dagger}$ \\ ${ }^{1}$ Université de Lorraine, LCP-A2MC, EA 3469, 1 Boulevard François Arago, Metz F-57078, France \\ ${ }^{2}$ Université Pierre et Marie Curie, UMR 8234 PHENIX, Paris, France \\ ${ }^{3}$ Department of Chemistry, University of Cambridge, Cambridge CB2 1EW, United Kingdom \\ ${ }^{4}$ Università di Trieste, Dipartimento di Fisica, strada Costiera 11, 34151 Grignano (Trieste), Italy
}

(Received 27 June 2015; published 28 October 2015)

\begin{abstract}
The structural and thermodynamic behavior of a deeply supercooled Lennard-Jones liquid, and its random first-order transition (RFOT) to an ideal glass is investigated, using a system of two weakly coupled replicas and the hypernetted chain integral equation for the pair structure of this symmetric binary system. A systematic search in the density-temperature plane points to the existence of two glass branches below a density-dependent threshold temperature. The branch of lower free energy exhibits a rapid growth of the structural overlap order parameter upon cooling and may be identified with the ideal glass phase conjectured by several authors for both spin and structural glasses. The RFOT, signaled by a sharp discontinuity of the order parameter, is predicted to be weakly first order from a thermodynamic viewpoint. The transition temperature $T_{\text {cr }}$ increases rapidly with density and approximately obeys a scaling relation valid for a reference system of particles interacting via a purely repulsive $1 / r^{18}$ potential.

DOI: 10.1103/PhysRevE.92.042316

PACS number(s): 64.70.Q-, 61.20.Gy, 05.20.Jj
\end{abstract}

\section{INTRODUCTION}

Upon quenching a liquid below the freezing temperature sufficiently rapidly to bypass crystallization, the microscopic dynamics of the supercooled liquid slow down dramatically. When the structural relaxation time becomes comparable to experimental time scales, the liquid undergoes a glass transition at a cooling-rate-dependent temperature $T_{\mathrm{g}}$, signaled by a rapid change in slope of measured thermodynamics quantities [1]; their extrapolation to lower temperatures hints at the vanishing of the configurational entropy $s_{\mathrm{c}}$ at the Kauzmann temperature $T_{\mathrm{K}}$ [2]. There is growing theoretical evidence that, near $T_{\mathrm{K}}$, the supercooled liquid will undergo an equilibrium thermodynamic transition to an "ideal glass," usually referred to as a random first-order transition (RFOT) [3], which is not directly observable experimentally because equilibration of the coexisting phases is only achieved after a divergent relaxation time.

The RFOT for continuous systems is reminiscent of a similar transition predicted by mean-field theory of spin glasses [3] and has been investigated within the replica formalism, using theoretical tools borrowed from statistical mechanics of liquids [4]. Mézard, Parisi, and co-workers used the hypernetted chain (HNC) free energy functional of the pair structure of an $m$-replica system, within an extension to a continuous number $m$ of replicas $[5,6]$. Their work predicts a second-order thermodynamic transition at a critical temperature $T_{\mathrm{cr}}$, signaled by a discontinuous jump of a structural order parameter. Our own investigations were based on a more pedestrian, but robust and physically transparent, implementation of the replica formalism, using a two-replica system [7] of "soft spheres" interacting via an inverse power

\footnotetext{
${ }^{*}$ Corresponding author: jean-marc.bomont@univ-lorraine.fr †jph32@cam.ac.uk

pastore@ts.infn.it
}

$\left(1 / r^{n}\right)$ pair potential with $n=12$ [8], and the same HNC closure, as well as the thermodynamically self-consistent integral equation of Rogers and Young (RY) [9]. Both HNC and RY closures predict a weakly first-order thermodynamic transition, but at a significantly different temperature $T_{\mathrm{cr}}$.

Systems involving inverse-power pair potentials satisfy a simple scaling property according to which the reduced excess thermodynamic properties depend only on the single variable $\Gamma=\rho^{*} /\left(T^{*}\right)^{3 / n}$ rather than on the reduced density and temperature separately. In the present paper we extend our investigations to the Lennard-Jones (LJ) 12-6 potential, which involves a short-range repulsion and a long-range attraction. The main objective of this work is to confirm that the RFOT scenario predicted in Ref. [8] carries over to the case where there are two independent thermodynamic variables, such that the single transition point for "soft spheres" $[5,6]$ turns into a line of first-order transitions in the $\left(\rho^{*}, T^{*}\right)$ plane. In the present paper, the numerical investigations are restricted to the HNC closure, which has the considerable advantage of allowing free energies to be calculated from the knowledge of pair correlation functions alone $[4,10]$. The same closure was used in the work of Parisi and co-workers [5,6]; moreover, the $\mathrm{HNC}$ closure is the natural starting point for higher-order approximations [4]. More accurate thermodynamically consistent integral equations pose severe numerical convergence problems at very low temperatures due to the attractive component of the pair interaction and are the subject of a future publication.

\section{PAIR STRUCTURE OF A SYMMETRIC $m$-REPLICA SYSTEM}

We consider a fully symmetric "mixture" of $m$ identical systems (or replicas), each containing $N$ atoms in a volume $V$, with number density $\rho_{\alpha}=\rho=N / V(1 \leqslant \alpha \leqslant m)$. Atoms inside each replica interact via the Lennard-Jones pair 
potential:

$$
v_{\alpha \alpha}(r) \equiv v(r)=4 \varepsilon\left[\left(\frac{\sigma}{r}\right)^{12}-\left(\frac{\sigma}{r}\right)^{6}\right], \quad 1 \leqslant \alpha \leqslant m,
$$

where $r$ is an interatomic distance, $\varepsilon$ sets the energy scale, and the atomic diameter $\sigma$ sets the length scale. The equilibrium state of each replica is characterized by the reduced variables $\rho^{*}=\rho \sigma^{3}$ and $T^{*}=k_{\mathrm{B}} T / \varepsilon$, where $k_{\mathrm{B}}$ is Boltzmann's constant and $T$ is the temperature. We use reduced distances $x=r / \sigma$ throughout. Atoms in different replicas interact via a weak, short-range attractive potential chosen to be $(1 \leqslant \alpha<\beta \leqslant m)$

$$
v_{\alpha \beta}(r) \equiv v_{12}(x)=-\varepsilon_{12} w(x)=-\varepsilon_{12}\left[\frac{c^{2}}{x^{2}+c^{2}}\right]^{6},
$$

where $c=0.3 \sigma$ is chosen such that the range of the attraction is significantly shorter than $\sigma$, to ensure that one atom of replica $\alpha$ can interact with at most one atom of replica $\beta$. For nonzero $\varepsilon_{12}$, the attraction (2) induces clustering of atoms of different replicas into polyatomic "molecules" at sufficiently low temperature. The precise shape of $w(x)$ is irrelevant, since we are eventually interested in the limit $\varepsilon_{12} \rightarrow 0$.

The total potential energy of $m$ interacting replicas reads

$$
\begin{aligned}
V_{m \times N}\left(\left\{\mathbf{x}_{i}^{\alpha}\right\}, 1 \leqslant \alpha \leqslant m\right)= & \sum_{\alpha=1}^{m} \sum_{j>i} v\left(\left|\mathbf{x}_{i}^{\alpha}-\mathbf{x}_{j}^{\alpha}\right|\right) \\
& +\frac{1}{2} \sum_{\alpha \neq \beta} \sum_{i, j} v_{12}\left(\left|\mathbf{x}_{i}^{\alpha}-\mathbf{x}_{j}^{\beta}\right|\right),
\end{aligned}
$$

where $\mathbf{x}_{i}^{\alpha}=\mathbf{r}_{i}^{\alpha} / \sigma$ is the reduced position vector of atom $i$ in replica $\alpha$. In view of the replica symmetry, the pair structure of the $m$-replica system is characterized by just two independent pair distribution functions and two independent direct correlation functions, namely,

$$
\begin{aligned}
& g_{\alpha \alpha}(x) \equiv g_{11}(x)=g(x), \quad c_{\alpha \alpha}(x) \equiv c_{11}(x)=c(x), \\
& g_{\alpha \beta}(x) \equiv g_{12}(x)=g^{\prime}(x), \quad c_{\alpha \beta}(x) \equiv c_{12}(x)=c^{\prime}(x) .
\end{aligned}
$$

The set of $m(m-1)$ Ornstein-Zernike (OZ) relations [4] between the total and direct correlation functions $h_{\alpha \beta}(x)=$ $g_{\alpha \beta}(x)-1$ and $c_{\alpha \beta}(x)$ reduces to just two coupled relations:

$$
h(x)=c(x)+\rho^{*} c(x) \otimes h(x)+(m-1) \rho^{*} c^{\prime}(x) \otimes h^{\prime}(x),
$$

$$
\begin{aligned}
h^{\prime}(x)= & c^{\prime}(x)+\rho^{*} c(x) \otimes h^{\prime}(x)+\rho^{*} c^{\prime}(x) \otimes h(x) \\
& +(m-2) \rho^{*} c^{\prime}(x) \otimes h^{\prime}(x),
\end{aligned}
$$

where $\otimes$ denotes a convolution product. Fourier transformation leads to the following expressions for $h(q)$ and $h^{\prime}(q)$ in terms of $c(q)$ and $c^{\prime}(q)$ (where $q=k \sigma$ is a reduced wave number):

$$
\begin{aligned}
h(q)= & \frac{1}{\Delta(q)}\left\{c(q)\left[1-c(q)-(m-2) c^{\prime}(q)\right]\right. \\
& \left.+(m-1) c^{\prime 2}(q)\right\}, \\
h^{\prime}(q)= & \frac{c^{\prime}(q)}{\Delta(q)},
\end{aligned}
$$

where

$$
\begin{aligned}
\Delta(q)= & {[1-c(q)]\left[1-c(q)+(m-2) c^{\prime}(q)\right] } \\
& -(m-1) c^{\prime 2}(q)
\end{aligned}
$$

and, e.g.,

$$
h(q)=\rho^{*} \int e^{i \mathbf{q} \mathbf{x}} h(x) d \mathbf{x} .
$$

Relations (6a) and (6b) must be supplemented by a closure relation. The present work is based on the HNC closure [4], involving the indirect correlation functions $\gamma(x)=h(x)-$ $c(x)$ and $\gamma^{\prime}(x)=h^{\prime}(x)-c^{\prime}(x)$ :

$$
\begin{aligned}
g(x) & =\exp [-\beta v(x)+\gamma(x)], \\
g^{\prime}(x) & =\exp \left[-\beta v_{12}(x)+\gamma^{\prime}(x)\right] .
\end{aligned}
$$

Knowledge of the pair functions allows the calculation of the thermodynamic properties of any of the $m$ replicas, for which the remaining $(m-1)$ replicas act like external fields. The excess internal energy per particle is hence given by

$$
\begin{aligned}
u^{e x}=\frac{U^{e x}}{N}= & 2 \pi \rho^{*} \int_{0}^{\infty} v(x) g(x) x^{2} d x \\
& +(m-1) 4 \pi \rho^{*} \int_{0}^{\infty} v_{12}(x) g^{\prime}(x) x^{2} d x .
\end{aligned}
$$

The pressure $P$ exerted by a single replica follows from the virial theorem

$$
\begin{aligned}
\frac{\beta P}{\rho}= & 1-\frac{2 \pi \rho^{*}}{3} \int_{0}^{\infty} \frac{\partial \beta v(x)}{\partial x} g(x) x^{3} d x \\
& -(m-1) \frac{4 \pi \rho^{*}}{3} \int_{0}^{\infty} \frac{\partial \beta v_{12}(x)}{\partial x} g^{\prime}(x) x^{3} d x,
\end{aligned}
$$

where $\beta=1 / k_{B} T$. A straightforward generalization of the derivation in Appendix B of Ref. [8] leads to the following expression for the excess chemical potential valid within $\mathrm{HNC}$ theory:

$$
\begin{aligned}
\beta \mu_{e x}= & \frac{\rho^{*}}{2} \int[h(x) \gamma(x)-2 c(x)] d \mathbf{x} \\
& +(m-1) \frac{\rho^{*}}{2} \int\left[h^{\prime}(x) \gamma^{\prime}(x)-2 c^{\prime}(x)\right] d \mathbf{x} .
\end{aligned}
$$

This expression does not depend explicitly on the pair potentials $v(x)$ and $v_{12}(x)$, and it remains valid in the limit $\varepsilon_{12} \rightarrow 0$. The dimensionless excess Helmholtz free energy then follows from the thermodynamic relation

$$
f_{e x}=\frac{\beta F_{e x}}{N}=\beta \mu_{e x}-\Pi_{e x},
$$

where $\Pi_{e x}=\beta P / \rho-1$. Iterative solutions of the two coupled integral equations obtained by combining the OZ relations (5) and the HNC closure relations (9), using Gillan's method [11], provide the pair structure of the $m$-replica system and the resulting thermodynamic properties for each state point $\left(\rho^{*}, T^{*}\right)$ and choice of the inter-replica coupling $\varepsilon_{12}$.

\section{SEARCH PROTOCOL FOR TWO-REPLICA SYSTEMS}

We henceforth restrict our attention to the two-replica system, along lines similar to those followed in our earlier 
work on "soft spheres" [8]. The key collective variable in the search for the RFOT is the overlap function [7]

$$
q_{1,2}\left(\left\{\mathbf{x}_{i}^{1}\right\},\left\{\mathbf{x}_{j}^{2}\right\}\right)=\frac{1}{N} \sum_{i=1}^{N} \omega\left(\left|\mathbf{x}_{i}^{1}-\mathbf{x}_{i}^{2}\right|\right),
$$

where $\omega(x)$ is an arbitrary function chosen such that $\omega(0)=$ 1 (corresponding to perfect overlap) and which vanishes rapidly for $x \gtrsim 1$. Since the function $w(x)$ in Eq. (2) satisfies precisely these conditions, we choose $\omega(x)=w(x)$. The order parameter for the RFOT is defined as the mean overlap:

$$
Q=\left\langle q_{1,2}\right\rangle=4 \pi \rho^{*} \int_{0}^{\infty} g^{\prime}(x) w(x) x^{2} d x .
$$

We examine the behavior of $Q\left(\rho^{*}, T^{*}, \varepsilon_{12}\right)$ in the limit $\varepsilon_{12} \rightarrow 0$. In the supercooled liquid, atoms eventually diffuse throughout the volume $V$ after a sufficiently long equilibration time so that the configurations of the two replicas are fully decorrelated, and $Q$ reduces to the random overlap value $Q_{r}$ as calculated from Eq. (15) with $g^{\prime}(x) \equiv 1$, i.e., $Q_{r}=$ $7 \pi^{2} \rho^{*} c^{3} / 128$. In the "ideal glass," on the other hand, atoms of both replicas remain localized near disordered equilibrium positions $\left\{\mathbf{X}_{i}\right\}$, so their configurations are highly correlated, and $Q$ takes on values $1 \gtrsim Q \gg Q_{r}$. The RFOT is hence expected to be characterized by a discontinuous jump of the order parameter in the thermodynamic limit [7]. A related structural signature of the RFOT is the zero separation value of the inter-replica correlation function $\Psi=h^{\prime}(x=0)$, which is trivially zero in the supercooled liquid only.

We have solved the coupled HNC integral equations for $g(x)$ and $g^{\prime}(x)$ along several isochores ranging from 1 to 1.4. For each $\rho^{*}$, and a given initial value $\varepsilon_{12}^{0}=0.001 \varepsilon$ of the inter-replica coupling, the temperature $T^{*}$ was gradually lowered to the point where the order parameters $\Psi$ and $Q$ undergo a discontinuous jump; the temperature at which the discontinuity occurs is referred to as $T_{d j}^{*}\left(\rho^{*}, \varepsilon_{12}\right)$. At this temperature $\varepsilon_{12}$ was then progressively lowered from the initial value $\varepsilon_{12}^{0}$, using a small decrement, until extinction $\left(\varepsilon_{12}=0\right)$, and the corresponding HNC solutions $g(x)$ and $g^{\prime}(x)$ were used to calculate the order parameters $Q$ and $\Psi$, as illustrated in Fig. 1.

Since $\Psi \neq 0$ and $Q>Q_{r}$ at the state point $\left(\rho^{*}, T_{d j}^{*}, \varepsilon_{12}=\right.$ 0 ), the system is considered to be in a glassy state. Starting from $T_{d j}^{*}$, a glass branch $G_{1}$ is subsequently mapped out upon gradually varying the temperature $T^{*}$ along the isochore, keeping $\varepsilon_{12}=0$. It is important to stress that, while $T_{d j}^{*}\left(\rho^{*}, \varepsilon_{12}^{0}\right)$ may depend on the choice of $\varepsilon_{12}^{0}$, the properties of the resulting glass branch $G_{1}$ are totally independent of that choice. Contrary to intuition, the order parameters $Q$ and $\Psi$ are systematically found to increase upon heating, up to a maximum temperature $T_{\mathrm{m} 1}^{*}$. At $T_{\mathrm{m} 1}^{*}$, the order parameters undergo a second discontinuous jump towards still larger values, corresponding to a new glass state $G_{2}$. If the temperature is now lowered, starting from the new state $G_{2}$, a second glass branch $G_{2}$ is generated, along which the order parameters $Q$ and $\Psi$ now increase, as expected for an ideal glass, where atoms are increasingly localized around the equilibrium positions $\left\{\mathbf{X}_{i}\right\}$. Note that the $G_{2}$ branch extends up to a slightly higher temperature $T_{m 2}^{*}>T_{m 1}^{*}$ than the $G_{1}$ branch, as seen in Fig. 2 .

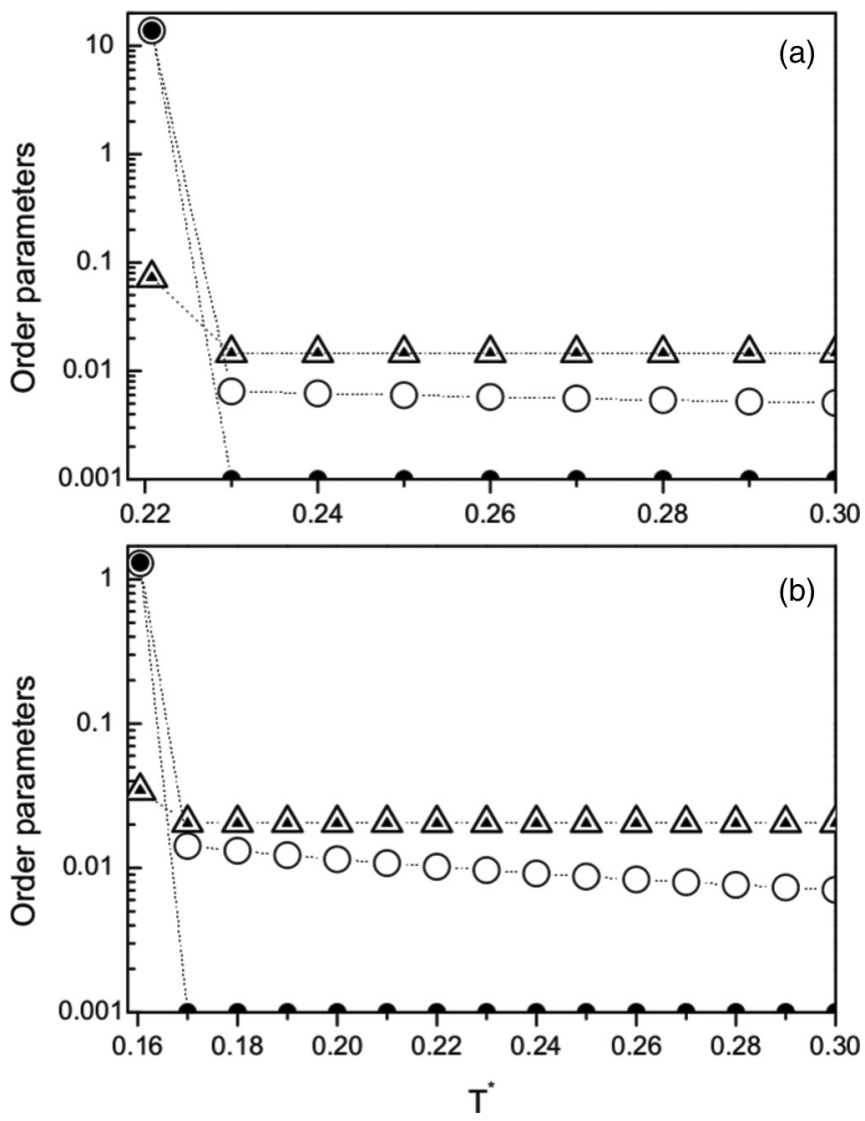

FIG. 1. Variation of the order parameters $\Psi$ (circles, shifted by 0.001 along the vertical axis) and $Q$ (triangles) as functions of $T^{*}$ for $\varepsilon_{12}^{0} / \varepsilon=0.001$ (open symbols) and once the coupling has been progressively sent to zero (solid symbols). In both cases a discontinuous jump of the order parameters is observed at (a) $T^{*}=T_{d j}^{*}=0.23$ for $\rho^{*}=1$ and (b) $T^{*}=T_{d j}^{*}=0.17$ for $\rho^{*}=1.4$.

No "glassy" solutions of HNC are found for $T^{*}>T_{m 1}^{*}$ along $G_{1}$, or for $T^{*}>T_{m 2}^{*}$ along $G_{2}$.

Our search protocol hence predicts the existence of three thermodynamic branches, namely $L$ (supercooled liquid), $G_{1}$, and $G_{2}$ upon varying the temperature $T^{*}$ along an isochore. The same scenario is observed along each isochore explored in this work and is qualitatively identical to that reported in our soft-sphere work [8]. Figure 2 shows the variation of $Q$ and $\Psi$ as functions of the dimensionless variable $\Gamma=\rho^{*} /\left(T^{*}\right)^{1 / 6}$ along the isochores $\rho^{*}=1$ and 1.4 (the relevance of the variable $\Gamma$ will become clearer in Sec. IV). The corresponding HNC pair distribution functions $g(x)$ and $g^{\prime}(x)$ are shown in Fig. 3 at the respective RFOT temperatures (to be determined in Sec. V). In each case, $g^{\prime}(x)$ exhibits a sharp central peak for both $G_{1}$ and $G_{2}$ states, but of much larger amplitude on the $G_{2}$ branch. The corresponding coordination number is invariably very close to 1 , as expected from the "pairing" of atoms of opposite replicas; this same mechanism explains why $g^{\prime}(x)$ nearly coincides with $g(x)$ on the $G_{2}$ branch as $x$ increases, since the atoms of the two replicas are localized close to the equilibrium positions $\left\{\mathbf{X}_{i}\right\}$. The oscillations of $g^{\prime}(x)$ on the $G_{1}$ branch are in phase with those of $g(x)$ but are much more rapidly damped, pointing to a lesser correlation between atomic positions of opposite replicas. As already argued in 

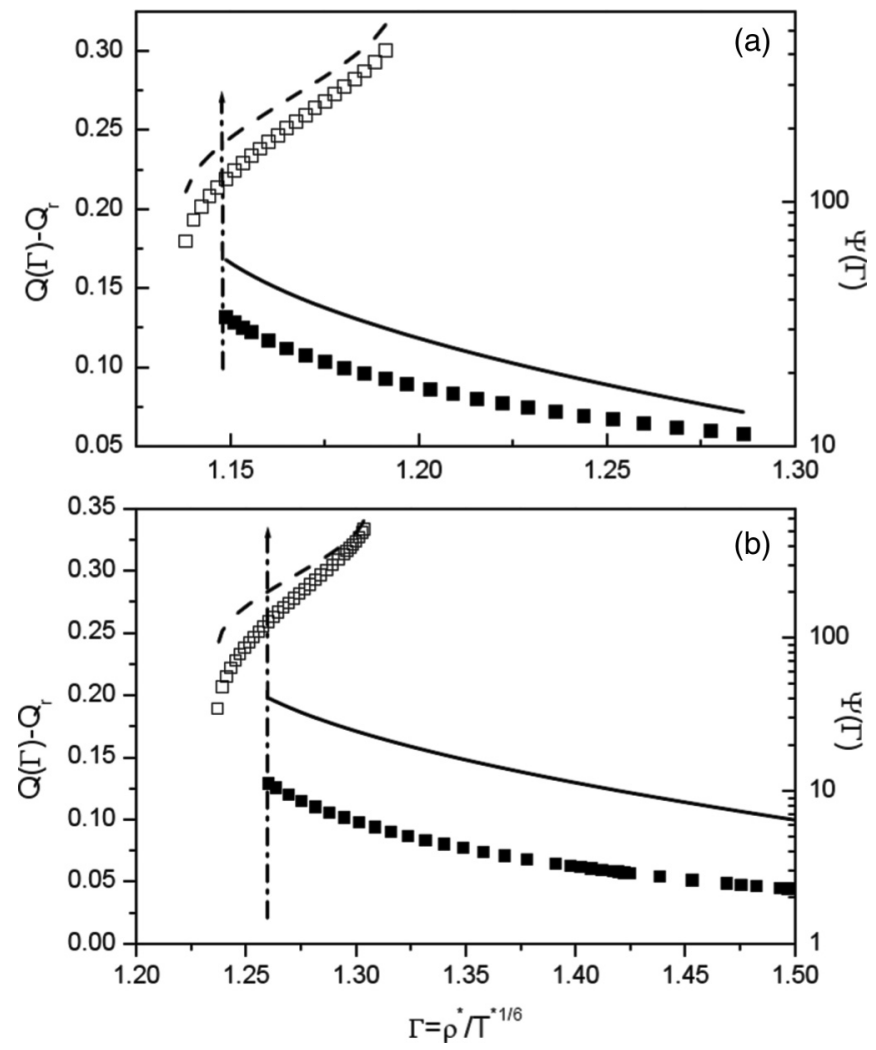

FIG. 2. Order parameters $Q(\Gamma)-Q_{r}$ (squares, left-hand axis) and $\Psi(\Gamma)$ (line, right-hand axis) vs $\Gamma=\rho^{*} /\left(T^{*}\right)^{1 / 6}$ along the two glass branches $G_{1}$ (solid squares and solid line) and $G_{2}$ (open squares and dashed line): (a) along the isochore $\rho^{*}=1$, where the discontinuity occurs at $\Gamma=\Gamma_{m 1}=1.15\left(T_{m 1}^{*}=0.43\right)$, and (b) along the isochore $\rho^{*}=1.4$, where the discontinuity occurs at $\Gamma=\Gamma_{m 1}=1.26\left(T_{m 1}^{*}=1.88\right)$.

Ref. [8], the different structural behavior along the two glass branches may be tentatively explained in terms of a rugged free energy surface [12] representing the functional $F[\rho(\mathbf{r})]$, where $\rho(\mathbf{r})$ is the local density, which reduces to the uniform density $\rho$ in the fully equilibrated liquid phase [3]. In the $G_{2}$ phase, which we identify with the ideal glass, the configurations are trapped in the lowest (noncrystalline) valley of the free energy landscape, while in the $G_{1}$ phase, atoms are trapped near slightly different equilibrium positions, corresponding to neighboring minima of the surface, which are separated by a free energy barrier; the latter is eventually overcome upon increasing the temperature so that the $G_{2}$ phase is finally reached.

\section{KAUZMANN TEMPERATURES}

We now return to the supercooled liquid $(L)$ phase corresponding to $\varepsilon_{12} \equiv 0$, i.e., to a single replica $(m=1)$. The objective is to calculate the line of Kauzmann temperatures $T_{\mathrm{K}}^{*}\left(\rho^{*}\right)$ at which the configurational entropy (or complexity) $s_{\mathrm{c}}$ vanishes along the different isochores under study. Within HNC theory, the excess free energy at each state point may be calculated from $g(x)$, using Eqs. (11)-(13), with $m=1$. Deep in the supercooled regime, atomic configurations $\left\{\mathbf{x}_{i}\right\}$ are trapped for very long times (of the order of the structural
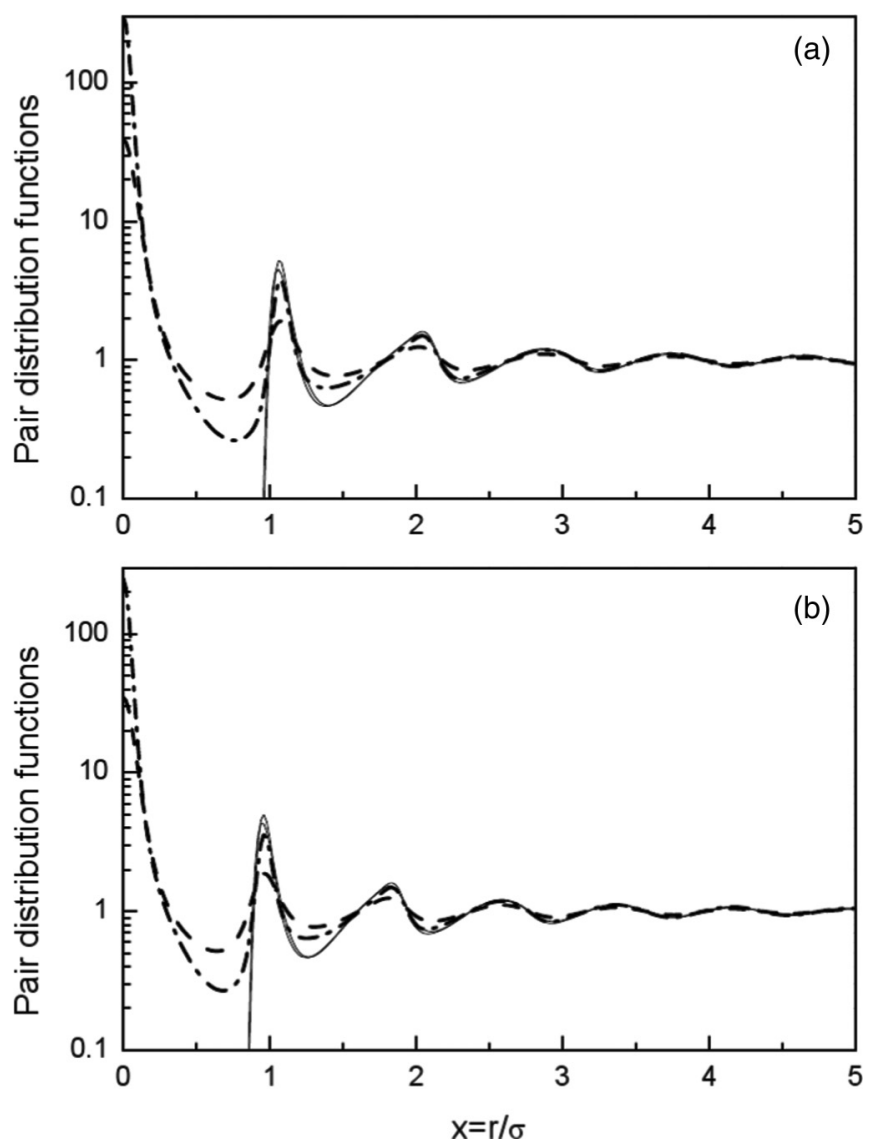

FIG. 3. HNC results for the inter-replica pair distribution function $g^{\prime}(x)$ at $T^{*}=T_{\mathrm{cr}}^{*}$. Dashed line, $G_{1}$ branch; dash-dotted line, $G_{2}$ branch. For comparison, $g(x)$ of the $G_{1}$ and $G_{2}$ branches is shown as a solid curve. All pair distribution functions are plotted on a logarithmic scale: (a) $\rho^{*}=1$, (b) $\rho^{*}=1.4$.

relaxation time) in local minima of the rugged free energy landscape, so that individual "caged" atoms vibrate around long-lived "equilibrium" positions $\left\{\mathbf{X}_{i}\right\}$. It is hence natural to divide the free energy and entropy into "configurational" (c) and "vibrational" $(v)$ contributions [5]:

$$
\begin{gathered}
f=\frac{\beta F}{N}=f_{i d}+f_{e x}=f_{c}+f_{\mathrm{v}}, \\
s=\frac{S}{N k_{B}}=s_{i d}+s_{e x}=s_{c}+s_{v} .
\end{gathered}
$$

Within a quasiharmonic "Einstein" approximation, each atom vibrates around its long-lived equilibrium position with a mean oscillation frequency $\omega_{0}$, and $f_{\mathrm{v}}$ is calculated from the partition function of a three-dimensional harmonic oscillator:

$$
f_{\mathrm{v}}=-\ln \left[\frac{1}{\Lambda^{3}}\left(\frac{2 \pi k_{B} T}{m \omega_{0}^{2}}\right)^{3 / 2}\right]
$$

where $\Lambda$ is the de Broglie thermal wavelength. The square of the mean vibration frequency is determined by the mean square force acting on an atom [4]:

$$
\omega_{0}^{2}=\frac{\rho}{3 m} \int \nabla^{2} v(r) g(r) d \mathbf{r} .
$$




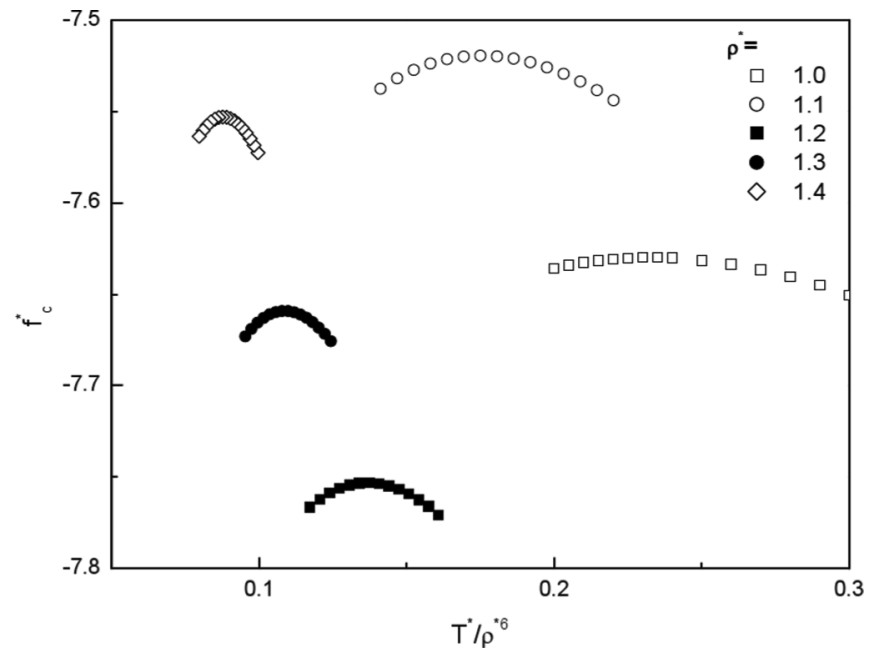

FIG. 4. Configurational free energy per particle $f_{c}^{*}$ of the $L$ phase as a function of $T^{*} /\left(\rho^{*}\right)^{6}$ along five isochores (shifted where indicated): $\rho^{*}=1,1.1,1.2$ (shifted by -1$), 1.3$ (shifted by -2.5 ), and 1.4 (shifted by -5 ).

Note that the classic expression (18) for $\omega_{0}$ also results from Mézard and Parisi's "small cage" expansion [5]. Combining Eqs. (16)-(18), and recalling that $f_{\text {id }}=\ln \left(\rho \Lambda^{3}\right)-1$, one arrives at

$$
f_{\mathrm{c}}\left(\rho^{*}, T^{*}\right)=f_{\mathrm{ex}}\left(\rho^{*}, T^{*}\right)+\ln \left[\rho^{*}\left(\frac{2 \pi T^{*}}{\omega_{0}^{* 2}}\right)^{3 / 2}\right]-1,
$$

where $\omega_{0}^{* 2}=m \omega_{0}^{2} \sigma^{3} / \varepsilon$ is the dimensionless vibration frequency.

The variation of the reduced configurational free energy $f_{\mathrm{c}}^{*}=F_{\mathrm{c}} / N \varepsilon=f_{\mathrm{c}} T^{*}$ with $T^{*} / \rho^{* 6}$ along five isochores is shown in Fig. 4. In each case, $f_{c}^{*}$ is seen to go through a maximum at a temperature $T_{\mathrm{K}}^{*}$ where the configurational entropy $s_{\mathrm{c}}=-\left(\partial f_{\mathrm{c}}^{*} / \partial T^{*}\right)_{\rho^{*}}$ vanishes (Kauzmann temperature). The values of $T_{\mathrm{K}}^{*}\left(\rho^{*}\right)$ are listed in Table I.

\section{RFOT ALONG ISOCHORES}

We now turn to the determination of the RFOT temperature $T_{\mathrm{cr}}^{*}\left(\rho^{*}\right)$ as a function of $\rho^{*}$. To that purpose we compare the total free energies of the $L, G_{1}$, and $G_{2}$ branches as functions of $T^{*}$ along some isochores. The HNC estimate of the free

TABLE I. Numerical values of the $\mathrm{LJ}$ characteristic temperatures $T_{\mathrm{K}}^{*}$ and $T_{\mathrm{cr}}^{*}$ vs reduced densities from the HNC approximation. ${ }^{(*)}$ : Comparison is made with the corresponding repulsive $1 / r^{18}$ potential model quantities, calculated with $\mathrm{HNC}$ at $\rho^{*}=1$.

\begin{tabular}{lclll}
\hline \hline$\rho^{*}$ & $T_{\mathrm{K}}^{*}$ & $\Gamma_{\mathrm{K}}$ & $T_{\mathrm{cr}}^{*}$ & \multicolumn{1}{c}{$\Gamma_{\mathrm{cr}}$} \\
\hline 1.00 & 0.23 & 1.277 & 0.385 & 1.175 \\
1.10 & 0.31 & 1.337 & 0.6 & 1.197 \\
1.20 & 0.41 & 1.392 & 0.9 & 1.221 \\
1.30 & 0.53 & 1.445 & 1.28 & 1.2475 \\
1.35 & 0.59 & 1.474 & 1.51 & 1.26 \\
1.40 & 0.66 & 1.50 & 1.78 & 1.272 \\
$1^{(*)}$ & & 1.41 & & 1.285 \\
\hline \hline
\end{tabular}
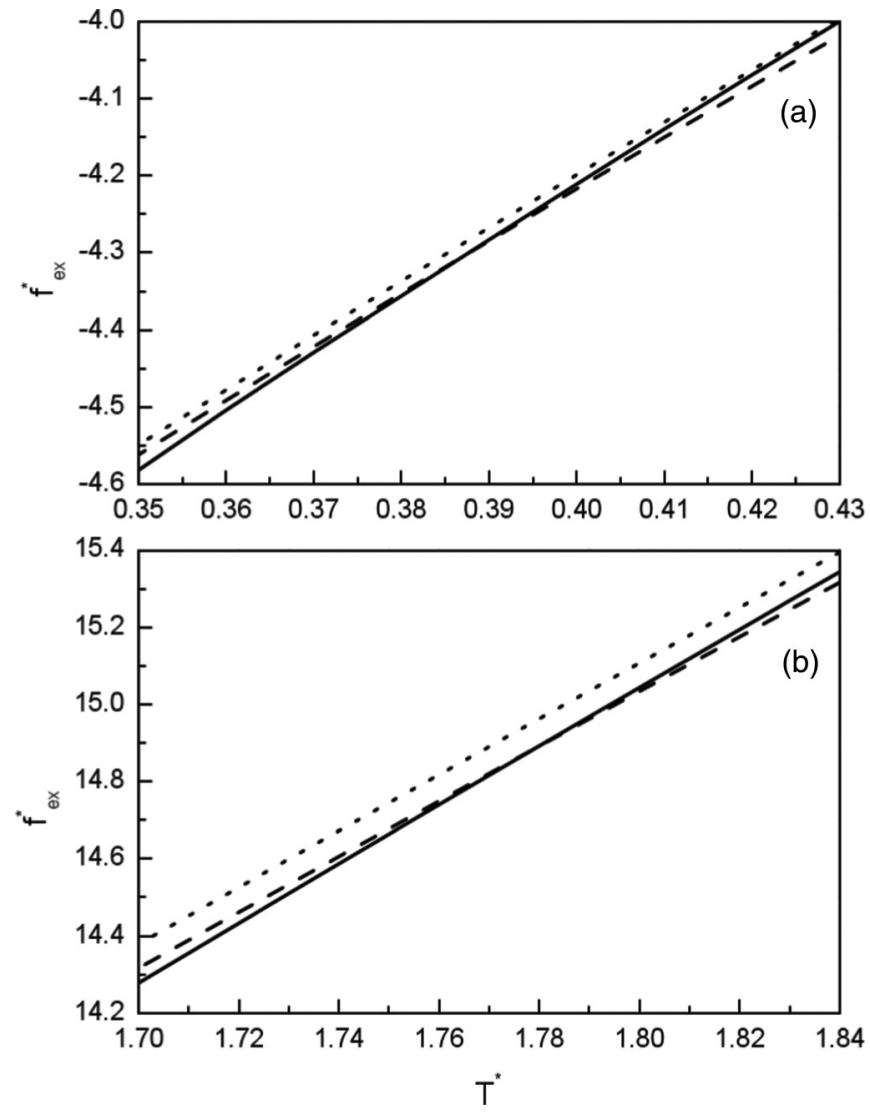

FIG. 5. Excess free energies $f_{e x}^{*}$ of the three branches $L$ (dashed curve), $G_{1}$ (dotted curve), and $G_{2}$ (full curve) as functions of $T^{*}$ along 2 isochores: (a) $\rho^{*}=1$ and (b) $\rho^{*}=1.4$.

energy of the $L$ phase is calculated as explained in Sec. IV, setting $\varepsilon_{12} \equiv 0$ and $m=1$. For the glass phases $G_{1}$ and $G_{2}$, we use the HNC results for a two-replica system $(m=2)$ in the limit $\varepsilon_{12} \rightarrow 0$. The resulting reduced excess free energies $f_{e x}^{*}=F_{e x} / N \varepsilon$ are plotted versus $T^{*}$ along two isochores in Fig. 5. The free energies of the $G_{1}$ phase are seen to lie systematically above those of the $L$ and $G_{2}$ phases and hence play no role in the following. The free energies of the $L$ and $G_{2}$ phases cross at temperatures $T_{\mathrm{cr}}^{*}\left(\rho^{*}\right)$ listed in Table I, and signal the RFOT, with the ideal glass $G_{2}$ being the stable phase for $T^{*}<T_{\mathrm{cr}}^{*}$ along each of the isochores. The determination of the $L-G_{2}$ phase coexistence lines is conveniently achieved by the Maxwell double-tangent construction on a plot of $f^{*}$ as a function of the reduced volume per atom $v^{*}=1 / \rho^{*}$ along an isotherm. Two examples are shown in Fig. 6. The relative volume changes $\Delta v^{*} / v_{L}^{*}=\left(v_{L}^{*}-v_{G 2}^{*}\right) / v_{L}^{*}$ have been found to be of the order of $1 \%$. HNC is seen to predict that the RFOT is a weakly first-order transition, in agreement with our earlier predictions for the soft-sphere model [8].

At this stage, it is worth noting that the free energy calculations for the $G_{1}$ and $G_{2}$ phases do not require one to systematically follow the search protocol described in Sec. III for each isochore. Once solutions of the HNC equations have been obtained for a given state point $\left(\rho^{*}, T^{*}\right)$ of the branches $G_{1}$ and $G_{2}$, glassy solutions for neighboring isochores are easily obtained by using the former solutions as initial guesses in the iterative procedure for small increments 

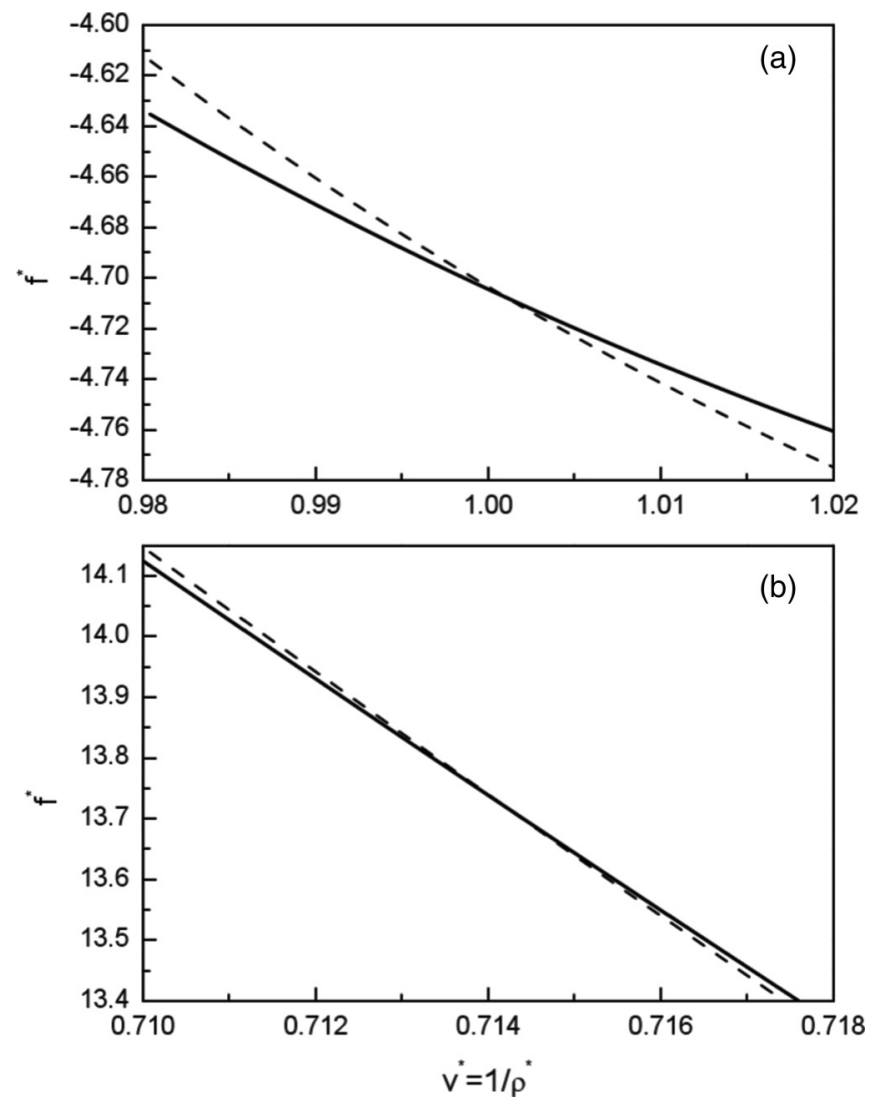

FIG. 6. Reduced free energies $f^{*}$ as functions of $v^{*}=1 / \rho^{*}$ for the $L$ (dashed curve) and $G_{2}$ (solid curve) phases at $T^{*}=T_{\text {cr }}^{*}$ along two isochores: (a) $\rho^{*}=1$ and (b) $\rho^{*}=1.4$.

$\Delta \rho^{*}$ in density, which greatly simplifies the detection of glassy states throughout the $\left(\rho^{*}, T^{*}\right)$ plane. We have checked that the resulting structures and thermodynamics are strictly identical to those obtained via the full search protocol of Sec. III at the required density.

The repulsive inverse-power pair potential $1 / r^{18}$, whose excess properties are uniquely characterized by a dimensionless coupling constant $\Gamma=\rho^{*} /\left(T^{*}\right)^{1 / 6}$, has been shown to accurately reproduce the structure and thermodynamics of LJ fluids at high densities ("hidden scale invariance") [13]. Table I shows that the values of $\Gamma_{\mathrm{cr}}=\rho^{*} /\left(T_{\mathrm{cr}}^{*}\right)^{1 / 6}$ appropriate for the supercooled LJ fluid at the five densities which were investigated become closer and closer to the value found for the $1 / r^{18}$ potential model within HNC theory, namely $\Gamma_{\mathrm{cr}} \simeq 1.285$, with increasing density.

Figure 7 compares the pair distribution functions $g(x)$ of the coexisting $L$ and $G_{2}$ phases at the respective RFOT temperatures $T_{\mathrm{cr}}^{*}\left(\rho^{*}\right)$ for three densities. When plotted as functions of $x^{\prime}=x\left(\rho^{*}\right)^{1 / 3}$, the pair distribution functions for each of the two phases are nearly indistinguishable, pointing to an underlying "universality" of the pair structure on both sides of the transition from the supercooled liquid to the ideal glass. In addition, the "hidden scale invariance" concept is very well illustrated here, since the $g(x)$ 's of the coexisting $L$ and $G_{2}$ phases at the RFOT of the repulsive $1 / r^{18}$ potential model are seen to coincide with those of the LJ potential model at $T_{\mathrm{cr}}^{*}\left(\rho^{*}\right)$.
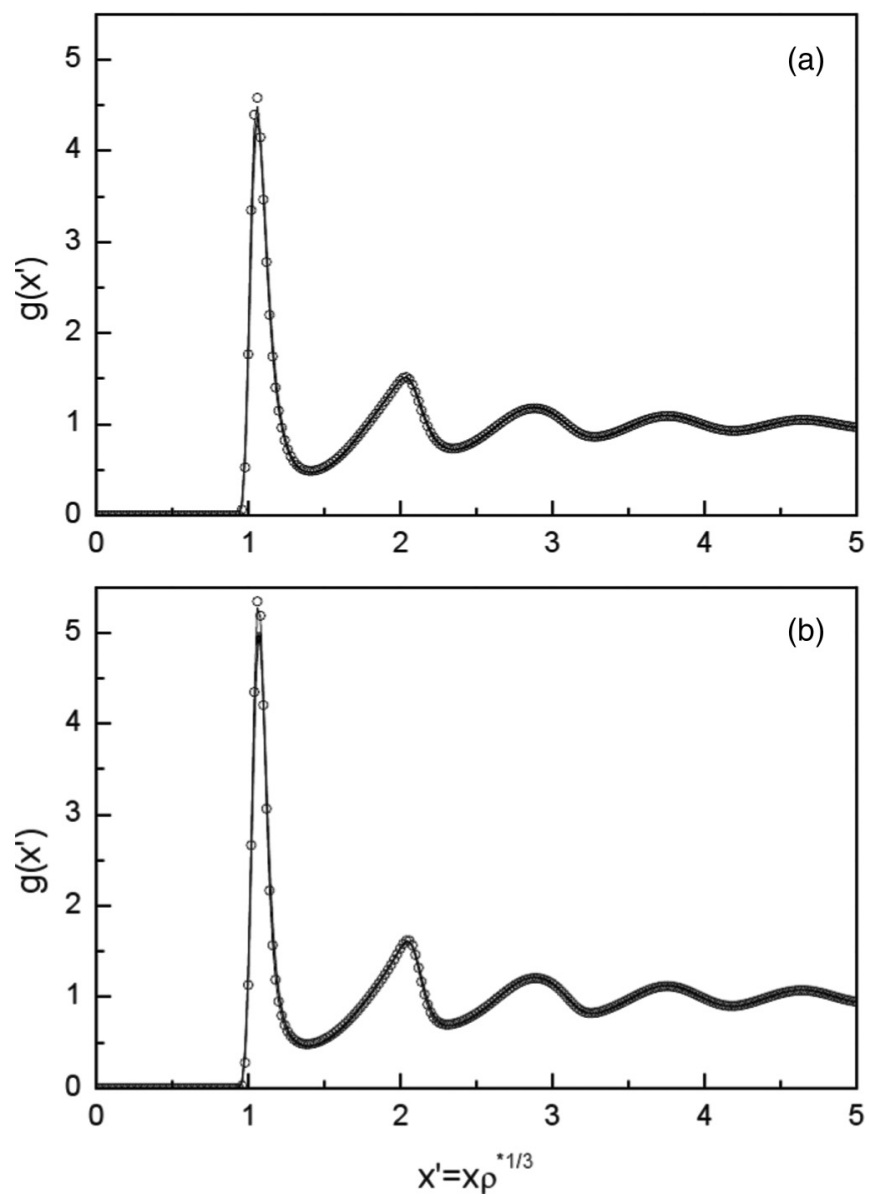

FIG. 7. Comparison of the LJ $g\left(x^{\prime}\right)$ at the RFOT $\left(T^{*}=T_{\mathrm{cr}}^{*}\right)$ as a function of $x^{\prime}=x\left(\rho^{*}\right)^{1 / 3}$ for $\rho^{*}=1,1.2$, and 1.4 (solid curves). $\mathrm{LJ}$ pair correlation functions are nearly indistinguishable from those of the repulsive $1 / r^{18}$ potential model at $\Gamma_{\text {cr }}=1.285$ (circles): (a) $L$ branch and (b) $G_{2}$ branch.

\section{CONCLUSION}

The results presented in this paper confirm our earlier predictions [8] for the random first-order transition from a deeply supercooled liquid to an ideal glass under the assumption that thermodynamic equilibrium has been reached. Our calculations are based on the two-replica formalism [7] and numerical solutions of the HNC integral equation for the pair structure of this binary system. While the earlier work was restricted to a soft-sphere model with a purely repulsive inverse-power pair potential (which implies simplifying scaling properties), we have explored the influence of a long-range attraction on the RFOT, by considering the more "realistic" Lennard-Jones potential. Adapting the search protocol to a system whose structural and thermodynamic properties now depend on two independent variables, we have been able to extract a line of weakly first-order transitions in the $\left(\rho^{*}, T^{*}\right)$ plane, characterized by a $1 \%$ relative volume change, and a large discontinuity of the two related structural order parameters, $Q$ and $\Psi$. The main lessons which may be drawn from the present work may be summarized as follows: 
(a) The two-replica approach, combined with the HNC integral equation, predicts the existence of two glass branches below density-dependent threshold temperatures $T_{\mathrm{m} 1}^{*}$ and $T_{\mathrm{m} 2}^{*}$. The branch of lowest free energy, $G_{2}$, may be identified with the hypothetical ideal glass phase. A possible free energy landscape interpretation of the metastable $G_{1}$ branch was put forward in Sec. III. The pertinence of the energy landscape concept for mean-field spin glasses appears to carry over to the HNC description of structural glasses, underlining the mean-field character of HNC theory, and more generally of classical density functional approximations, which account for strong interatomic correlations but cannot handle thermal fluctuations correctly [14]. The existence of these two glass branches appears to be a robust finding, since it is predicted both for systems with purely repulsive interactions [8] and in the presence of a long-range attraction, as in the LJ pair potential examined here.

(b) Table I shows that the RFOT temperature $T_{\mathrm{cr}}^{*}$ is found to be higher than the Kauzmann temperature $T_{K}^{*}$, in contradiction with the predictions of Parisi and co-workers [5,6] which imply that $T_{\mathrm{cr}}^{*}=T_{K}^{*}$ and that the RFOT is a second-order thermodynamic transition, as opposed to the weakly first-order character predicted in Ref. [8] and in the present work. This disagreement may be due to the fact that we compare the free energy of a single-replica system, describing the fully equilibrated supercooled liquid phase with the lowest free energy calculated from a two-replica system in the limit of vanishing inter-replica coupling, which corresponds to the glass phase $G_{2}$ characterized by finite values of the order parameters $\Psi$ and $Q-Q_{r}$. In other words we anticipate a priori the possible existence of two free energy branches which intersect at $T_{\mathrm{cr}}^{*}$ just as liquid and crystal free energy curves intersect near freezing [15]. The HNC results confirm this scenario. In our earlier work on soft spheres [8], the HNC closure also predicted $T_{\text {cr }}^{*}>T_{K}^{*}$; however, the thermodynamically self-consistent RY closure [9] predicted $T_{\mathrm{cr}}^{*}=T_{K}^{*}$ within numerical uncertainties, suggesting that the HNC prediction reflects the insufficiencies of that closure at high densities. (c) The pertinence of the "hidden scale invariance" concept of Dyre and co-workers [13] is confirmed, since the thermodynamic and structural properties of supercooled and glassy states of the LJ fluid are reasonably well represented by a reference system of atoms interacting via a repulsive $1 / r^{18}$ pair potential. Note, however, that the Kauzmann temperatures $T_{\mathrm{K}}^{*}\left(\rho^{*}\right)$ deviate significantly from this scale invariance as is clear from Fig. 4 and Table I. This is perhaps not too surprising, since $T_{\mathrm{K}}^{*}$ is not a genuine thermodynamic property.

(d) Along similar lines our HNC calculations point to a striking "universality" of the pair structure of the $L$ and $G_{2}$ phases at the RFOT temperature $T_{\mathrm{cr}}^{*}\left(\rho^{*}\right)$ (cf. Fig. 7).

We are planning two extensions of the present work. In Ref. [8], the HNC results for soft spheres were tested by using the more accurate, thermodynamically consistent RY integral equation [9]. The scenarios predicted by the two integral equations were shown to be identical although the RY values of $T_{\mathrm{K}}^{*}$ and $T_{\mathrm{cr}}^{*}$ lie significantly below those predicted by HNC. We are carrying out a similar test in the case of the LJ system, using improved closure relations $[16,17]$. Preliminary results confirm once more the HNC scenario qualitatively with significant quantitative differences, which will be reported elsewhere.

A second extension of the present work is to switch from two to $m$ replicas (in particular $m=3$ ). The generalized expressions for the structural dynamics and thermodynamics of symmetric $m$-replica systems are reported in Sec. II. It is expected that the ideal glass branch $G_{2}$ will be recovered but there may be $(m-2)$ additional, metastable branches on top of the $G_{1}$ branch observed with the two-replica system, providing additional support to the interpretation of metastable glass branches in terms of the underlying free energy landscape.

\section{ACKNOWLEDGMENTS}

J.P.H. acknowledges a stimulating discussion with Patrick Charbonneau. J.M.B. would like to thank J. C. Dyre for useful information.
[1] P. G. Debenedetti and F. H. Stillinger, Nature (London) 410, 259 (2001).

[2] A. W. Kauzmann, Chem. Rev. 43, 219 (1948).

[3] For a collection of reviews of recent work, see Structural Glasses and Supercooled Liquids: Theory, Experiment and Applications, edited by P. G. Wolynes and V. Lubchenko (Wiley, Hoboken, NJ, 2012); see also L. Berthier and G. Biroli, Rev. Mod. Phys. 83, 587 (2011).

[4] J.-P. Hansen and I. R. McDonald, Theory of Simple Liquids, 4th ed. (Academic Press, Amsterdam, 2013); J.-M. Bomont, Adv. Chem. Phys. 139, 1 (2008).

[5] M. Mézard and G. Parisi, J. Phys. A: Math. Gen. 29, 6515 (1996); J. Chem. Phys. 111, 1076 (1999).

[6] B. Coluzzi, G. Parisi, and P. Verrochio, J. Chem. Phys. 112, 2933 (2000).

[7] S. Franz and G. Parisi, Phys. A (Amsterdam, Neth.) 261, 317 (1998).
[8] J.-M. Bomont, J.-P. Hansen, and G. Pastore, J. Chem. Phys. 141, 174505 (2014); 142, 107105 (2015); J.-M. Bomont and G. Pastore, Mol. Phys. 113, 2770 (2015).

[9] F. J. Rogers and D. A. Young, Phys. Rev. A 30, 999 (1984).

[10] T. Morita and K. Hiroike, Prog. Theor. Phys. 25, 537 (1961).

[11] M. J. Gillan, Mol. Phys. 38, 1781 (1979).

[12] P. Charbonneau, J. Kurchan, G. Parisi, P. Urbani, and F. Zamponi, Nat. Commun. 5, 3725 (2014).

[13] N. P. Bailey, U. R. Pedersen, N. Gnan, T. B. Schroder, and J. C. Dyre, J. Chem. Phys. 129, 184507 (2008).

[14] R. Evans, in Fundamentals of Inhomogeneous Fluids, edited by D. Henderson (Marcel Dekker, New York, 1991).

[15] Y. Singh, J. P. Stoessel, and P. G. Wolynes, Phys. Rev. Lett. 54, 1059 (1985).

[16] G. Zerah and J.-P. Hansen, J. Chem. Phys. 84, 2336 (1986).

[17] D. M. Duh and D. Henderson, J. Chem. Phys. 104, 6742 (1996). 